\title{
Athletes' level of knowledge and concern regarding osteoporosis
}

\author{
R. N. Palmer ${ }^{1}$ and H. E. Theobald ${ }^{2}$ \\ ${ }^{1}$ Nutritional Sciences Division, Faculty of Health and Medical Sciences, University of Surrey, Guildford GU2 7XH, \\ UK and ${ }^{2}$ GlaxoSmithKline Nutritional Healthcare Future Group, Brentford TW8 9GS, UK
}

During weight-bearing physical activity, bone is exposed to high levels of mechanical stress which frequently exceed normal function and can cause stress fractures. Bone fracture is a frequent cause of injury in competitive and recreational athletes ${ }^{(1)}$, therefore, a strong skeletal framework is vital for athletes to enable them to train and compete at their optimum. Furthermore, individuals who have had a previous bone fracture may be at an increased risk of further fracture and osteoporosis later in life ${ }^{(2)}$. However, there is limited data on whether osteoporosis is a concern for athletes.

Athletes' knowledge, attitudes and beliefs towards bone health were assessed in an on-line questionnaire (SurveyMonkey, Palo Alto, CA, USA). An e-mail invitation to participate was sent to 237 male and female athletes aged 18-60 years, who were members of the Lucozade Sport High Performance Panel and various recreational sports clubs in the UK and had previously taken part in GlaxoSmithKline research. The survey consisted of demographic questions $(n 4)$, multiple-choice questions $(n 20)$, ranking questions $(n$ 5) and open-ended questions ( $n$ 2). Of the fifty-two responses received, a total of fifty-one (thirty-five males and sixteen female) athletes (mean age 33.8 \pm 9.9 years SD) completed sufficient amount of the survey to be included in the analysis. Subjects participated in a wide variety of sports (twenty-two sports cited, of which fourteen were classed as weight bearing).

Athlete's knowledge of osteoporosis and the associated risk factors were assessed over seven questions. These questions examined knowledge by assessing ability to define osteoporosis, beliefs regarding the seriousness of the condition and the influence of personal behaviour and diet on susceptibility to the bone disorder. Participants were asked to assess whether they believed that a certain risk factor/ behaviour (i.e. ageing, female gender, male gender, smoking, regular sunlight exposure, going through/having been through the menopause, low $\mathrm{Ca}$ intake throughout life, high alcohol intake and extreme weight-bearing exercises) either definitely increases, probably increases, probably does not increase or definitely does not increase risk of bone loss. Alternatively they could respond that they did not know. Participants were also asked to answer 'true', 'false' or 'not sure' to an additional list of eight statements relating to osteoporosis risk factors and symptoms. Subjects scored a point for each correct response given.

Out of a maximum score of 22, the mean score for osteoporosis knowledge was $16.8 \pm 2.69$ sE. Nearly all participants stated that they knew what osteoporosis is and $96.0 \%$ were able to define it correctly. A total of $44.0 \%$ of athletes believed that low Ca intakes throughout life definitely increases risk of osteoporosis, while $43.1 \%$ responded that regular sunlight exposure probably does not increase risk and a further $37.3 \%$ that it definitely does not increase risk of the disease. In contrast, $15.7 \%$ of athletes believed that extreme weight-bearing exercise definitely increases osteoporosis risk. Only $15.7 \%$ of participants were concerned regarding their risk of bone loss; however $90.2 \%$ believed bone loss to be serious in relation to their health and well-being.

Overall, the athletes were found to have moderate knowledge of bone health, findings that compare with those of other studies in athletes ${ }^{(3,4)}$; however, they were not concerned about their risk of osteoporosis. This lack of concern could translate into a lack of protective behaviours for bone health and increase risk of osteoporosis later in life. These findings, therefore, suggest that athletes would benefit from further education on their susceptibility to osteoporosis, the seriousness of the disease as well as the importance of good nutrition and activity patterns as a means of reducing risk.

1. Arendt EA (2000) Clin Orthop Relat Res 372, 131-138.

2. Klotzbuecher CM, Ross PD, Landsman PB et al. (2000) J Bone Miner Res 15, 721-739.

3. Frederick L \& Hawkins ST (1992) J Am Diet Assoc 92, 299-305.

4. Turner LW \& Bass MA (2001) Int J Sport Nutr Exerc Metab 11, 482-489. 